\title{
Study of the bacteriological profile of neonatal septicemia and associated risk factors
}

\author{
Amrutha Kumari B. ${ }^{1}$, Vedavati B. I. ${ }^{2,}$ \\ ${ }^{1}$ Professor, Dept. of Microbiology, Mysore Medical College and Research Centre, Mysore, Karnataka, ${ }^{2}$ Tutor, Dept. of \\ Microbiology, Shimoga Institute of Medical Science, Shimoga, Karnataka, India
}

*Corresponding Author:

Email: drbivedu@gmail.com

\begin{abstract}
Introduction: Neonatal sepsis is one of the major causes of morbidity and mortality among the newborns in the developing countries. For the effective management of neonatal sepsis, knowledge about causative agent and antibiotic sensitivity pattern play a vital role. Present study was undertaken to analyze the various risk factors of bacterial sepsis, to isolate aerobic bacteria causing neonatal septicemia and to study the antibiogram of bacterial isolates.

Material and Methods: Study was done over a period of one year. 198 clinically suspected cases of neonatal septicemia were included in the study. Detailed record of various predisposing neonatal factors were noted in a pro-forma. Blood samples from the neonates were subjected to aerobic blood cultures. The identification of the causative organism was carried out by standard identification tests and antibiotic sensitivity testing was done as per Clinical and Laboratory Standards Institute guidelines.

Results: Of 198 neonates studied, predisposing factors for neonatal sepsis were mainly low birth-weight and preterm. Blood cultures were positive in $128(64.64 \%$ ) cases. Predominant organisms isolated were Staphylococcus aureus (54.7\%) and Klebsiella spp. (14.1\%). Among Staphylococcus aureus, 43(61.42\%) isolates were MRSA and most of them were sensitive only to vancomycin and linezolid. Most of the MSSA were sensitive to cotrimoxazole, clindamycin, ciprofloxacin, vancomycin, linezolid. Most of the Gram negative bacilli were sensitive to imipenem, piperacillin-tazobactam and amikacin.

Conclusion: For the effective management of neonatal sepsis continued surveillance is mandatory for each center due to temporal changes in the causative organisms and their antibiotic susceptibility.
\end{abstract}

Keywords: Bacteriological Profile, Neonatal Septicemia, Risk Factors, Antibiogram and Blood Culture.

\section{Introduction}

Neonatal sepsis is a major cause of neonatal mortality and morbidity in India. ${ }^{1}$ It is defined as "a clinical syndrome characterized by systemic signs and symptoms and bacteremia during the first month of life". ${ }^{2}$ It is divided into early-onset sepsis (EOS) and lateonset sepsis (LOS) on basis of timing of infection and presumed mode of transmission. ${ }^{3}$

Rates of infection vary by geographic area, resource endowment, maternal and infant risk factors. ${ }^{4}$ The incidence of neonatal bacterial sepsis varies from 1 to 4/1000 live births in developed countries, with considerable fluctuation over time and with geographic variation. ${ }^{5}$ In Asia the incidence of neonatal septicemia varies from 7.1 to $38 / 1000$ live births and in India it is $30 / 1000$ live births. ${ }^{6,7}$

The different predisposing neonatal factors are sex, gestational age, birth weight, out born or inborn status of babies and maternal factors like illness during pregnancy, prolonged rupture of membranes (>18 hrs) and difficult labor with instrumentation. ${ }^{6,8}$ Preterm and low birth weight infants especially very low birth weight (VLBW) have a 3 to 10 fold higher incidence of infection than full term neonates. ${ }^{9}$

The causative agents of neonatal septicemia changes in different geographical areas and from time to time. In India, Klebsiella pneumoniae is the most important causative agent followed by Staphylococcus aureus. In developed nations, Group B Streptococcus is responsible for the leading cause of neonatal septicemia. ${ }^{10}$ Other pathogens are Gram-negative enteric organisms, especially Escherichia coli, Listeria monocytogenes, other Streptococci (including the Enterococci), anaerobes and Haemophilus influenza. ${ }^{11,12}$

Periodic surveillance should be conducted in a particular setting to identify leading cause for neonatal septicemia and also to detect antibiotic sensitivity patterns. It is essential for appropriate management of neonal septicemia. Therefore the aims and objectives of the present study is to know the risk factor associated with septicemia in neonates, to isolate aerobic bacteria causing neonatal septicaemia, to study the antibiogram of bacterial isolates.

\section{Material and Methods}

Source of Data: The present study; "Study of the bacteriological profile of neonatal septicemia and associated risk factors" was carried out in Department of Microbiology, MMC \& RI, Mysore over a period of one year from 1st Jan 2014 - 31st Dec 2014.

\section{Method of collection of data}

Sample size: 198 clinically suspected cases of neonatal septicemia.

Inclusion criteria: Neonates admitted with signs and symptoms of septicemia.

Exclusion criteria: Neonates with no clinical evidence of sepsis. 
A detailed record of the various predisposing neonatal factors like sex, gestational age, birth weight, outborn or inborn status of babies and maternal factors like illness during pregnancy, prolonged rupture of membranes(PROM) $(>18$ hrs) and difficult labor with instrumentation were recorded in a pro-forma..

Collection of samples and processing: After obtaining informed consent from guardian, 1-2 ml blood was collected aseptically from neonates with suspected sepsis and inoculated into brain heart infusion broth. Broth was incubated at $37^{\circ}$ C..$^{13,6}$ After $6-18$ hours of incubation first subcultures were done by aseptically removing a few drops of the well-mixed medium and inoculating onto blood agar, chocolate agar and Mac Conkey agar plate. The blood agar and Mac Conkey agar plates were incubated aerobically at $37^{\circ} \mathrm{C}$. Chocolate agar plate was incubated in $5 \%$ to $10 \%$ carbon dioxide jar at $35^{\circ} \mathrm{C}$ for 48 hours. Growth was further processed and identified according to standard protocols. If no growth was detected then further subculture were performed on 2nd, 3rd and 7th day of incubation. Again growth was processed and identified as mentioned above. ${ }^{14,15}$

\section{Antibiotic susceptibility testing}

Antimicrobial susceptibility testing of isolates was done on Muller-Hinton agar by Kirby-Bauer disc diffusion method, according to the Clinical and Laboratory Standards Institute (CLSI) guidelines. ${ }^{16}$ In the present study the susceptibility testing was carried out using the following antibiotics: ampicillin (AMP), amoxyclav (AC), cefuroxime(CU), cefotaxime(CE), ceftriaxone $(\mathrm{CI})$, co-trimoxazole $(\mathrm{CO})$, clindamycin(CD), erythromycin(E), gentamicin $(G)$, high level gentamicin (HLG), ciprofloxacin(CF), vancomycin(VA), linezolid (LZ), imipenem (IMP), piperacilllin-tazobactam (PT), $\operatorname{amikacin}(\mathrm{AK})$ and aztreonam (AO).

Detection of MRSA (Methicillin resistant staphylococcus aureus) and MRCONS (methicillin resistant coagulase negative staphylococci) was done using $30 \mu \mathrm{g}$ cefoxitin disc on Muller Hinton agar plate. ${ }^{7}$ All the antibiotic disks were procured commercially from Hi-Media laboratories Pvt. Ltd. Mumbai. The diameter of the zone of inhibition was measured and interpreted according to CLSI guidelines.

\section{Results and Analysis}

The present study was carried out in the Department of Microbiology, Mysore Medical College and Research Institute, Mysore from January 2014 - December 2014. The observations made from the study are shown in following tables.

Table 1: Characteristics of neonates in neonatal sepsis

\begin{tabular}{|l|c|c|}
\hline \multicolumn{1}{|c|}{ Neonates } & N & \% \\
\hline Type of Sepsis & & \\
\hline EOS(0-3 days ) & 109 & 55.05 \\
\hline LOS(4-28days) & 89 & 44.94 \\
\hline Gender & & \\
\hline Male & 126 & 63.63 \\
\hline Female & 72 & 36.36 \\
\hline Gestation & & \\
\hline Preterm (PT) & 78 & 39.39 \\
\hline Full term(FT) & 120 & 60.60 \\
\hline Birth Weight (gms) & & \\
\hline Very low birth weight $(<1500)$ & 40 & 20.20 \\
\hline Low birth weight(1500-2500) & 89 & 44.94 \\
\hline Normal(> 2500) & 69 & 34.84 \\
\hline
\end{tabular}

Of 198 neonates studied, 109(55.05\%) belongs to early onset sepsis and 89(44.94\%) belongs to late onset sepsis. 126 were males, 72 were females. 78 babies were preterm and remaining were full term. $40(20.20 \%)$ neonates were VLBW(Very Low Birth Weight), 89(44.94\%) were LBW(Low birth weight) and remaining were normal weight.

Table 2: Risk factors in blood culture positive cases

\begin{tabular}{|l|c|c|}
\hline \multicolumn{1}{|c|}{ Risk Factors } & N & \% \\
\hline LBW & 85 & 66.40 \\
\hline PT & 58 & 45.31 \\
\hline $\begin{array}{l}\text { PROM(prolonged rupture } \\
\text { of membrane) }\end{array}$ & 20 & 15.62 \\
\hline BA(Birth asphyxia) & 20 & 15.62 \\
\hline Out born delivery & 12 & 9.30 \\
\hline
\end{tabular}




\begin{tabular}{|l|c|c|}
\hline Instrumental delivery & 12 & 9.30 \\
\hline Maternal fever & 2 & 1.50 \\
\hline Meconium stained liquor & 4 & 3.10 \\
\hline
\end{tabular}

Of 128 culture positive cases, most common risk factor was low birth weight followed by preterm, PROM and birth asphyxia.

Table 3: Organisms isolated in neonatal sepsis

\begin{tabular}{|l|c|c|c|c|c|c|}
\hline \multirow{2}{*}{ Organisms } & \multicolumn{4}{|c|}{ Types of sepsis } & \multicolumn{2}{c|}{} \\
\cline { 2 - 7 } & \multicolumn{2}{|c|}{ EOS } & \multicolumn{2}{c|}{ LOS } & \multicolumn{2}{c|}{ Total } \\
\cline { 2 - 7 } & $\mathbf{n}$ & $\mathbf{\%}$ & $\mathbf{n}$ & $\mathbf{\%}$ & $\mathbf{n}$ & $\mathbf{\%}$ \\
\hline Staphylococcus aureus & 37 & 55.22 & 33 & 54.00 & 70 & 54.70 \\
\hline $\begin{array}{l}\text { CONS(coagulase } \\
\text { negative staphylococcus) }\end{array}$ & 2 & 2.98 & 5 & 8.19 & 7 & 5.50 \\
\hline Enterococcus spp. & 8 & 11.94 & 2 & 3.27 & 10 & 7.80 \\
\hline Klebsiella spp. & 7 & 10.44 & 11 & 18.03 & 18 & 14.10 \\
\hline Enterobacter spp. & 1 & 1.49 & 2 & 3.27 & 3 & 2.30 \\
\hline Citrobacter spp. & 0 & 0 & 1 & 1.63 & 1 & 0.80 \\
\hline Acinetobacter spp. & 3 & 4.47 & 3 & 4.91 & 6 & 4.70 \\
\hline Candida spp. & 9 & 13.43 & 4 & 6.55 & 13 & 10.20 \\
\hline Total & 67 & 100 & 61 & 100 & 128 & 100 \\
\hline
\end{tabular}

Graph 1: Organisms isolated in culture positive cases

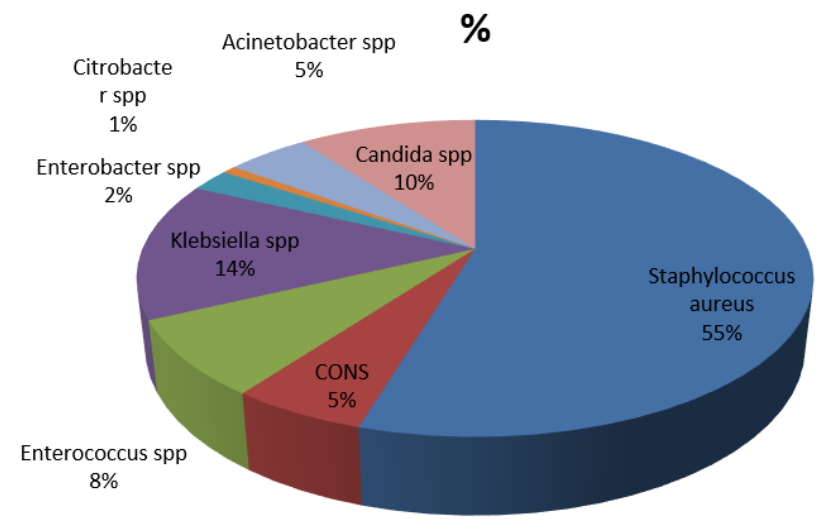

Among 198 cases studied 128 (64.64\%) were blood culture positive. In clinically suspected EOS cases $67(61.46 \%)$ were blood culture positive and in LOS $61(68.53 \%)$ were blood culture positive. Predominant organisms isolated were Staphylococcus aureus $(54.7 \%)$ and Klebsiella spp. (14.1\%) followed by Candida spp. (10.2\%).Predominant organisms isolated in EOS were S.aureus, Candida spp., Enterococcus and Kebsiella spp. In LOS it was S.aureus, Kebsiella spp. and CONS.

Table 4: Sensitivity pattern of gram positive bacterial isolates

\begin{tabular}{|l|c|c|c|c|c|}
\hline Organisms & $\begin{array}{c}\text { MSSA } \\
(\boldsymbol{\%})\end{array}$ & $\begin{array}{c}\text { MRSA } \\
(\mathbf{\%})\end{array}$ & $\begin{array}{c}\text { MSCONS } \\
(\boldsymbol{\%})\end{array}$ & $\begin{array}{c}\text { MRCONS } \\
(\boldsymbol{\%})\end{array}$ & $\begin{array}{c}\text { Enterococcus } \\
\text { spp. } \mathbf{( \% )}\end{array}$ \\
\hline Numbers & 27 & 43 & 5 & 2 & 10 \\
\hline AMP & $2(7.40)$ & 0 & $2(40.00)$ & 0 & 0 \\
\hline CO & $14(51.90)$ & $14(32.60)$ & $3(60.00)$ & 0 & - \\
\hline CD & $19(70.40)$ & $24(55.80)$ & $5(100.00)$ & $1(50.00)$ & - \\
\hline E & $12(44.40)$ & $12(27.90)$ & $3(60.00)$ & 0 & $3(30.00)$ \\
\hline G & $13(48.10)$ & $20(46.50)$ & $3(60.00)$ & $1(50.00)$ & - \\
\hline HLG & - & - & - & - & $2(80.00 \%)$ \\
\hline CF & $13(48.10)$ & $7(16.27)$ & 3 & 0 & 0 \\
& & & $(60.00)$ & & $10(100.00)$ \\
\hline VA & $27(100.00)$ & $43(100.00)$ & $5(100.00)$ & $2(100.00)$ & $10(100.00)$ \\
\hline LZ & $27(100.00)$ & $43(100.00)$ & $5(100.00)$ & $2(100.00)$ & 1 \\
\hline
\end{tabular}


MSSA- Methicillin sensitive staphylococcus aureus

MSCONS- Methicillin sensitive coagulase negative staphylococcus

Table 5: Sensitivity pattern of gram negative bacterial isolates

\begin{tabular}{|l|c|c|c|c|}
\hline Organisms & $\begin{array}{c}\text { Klebsiella } \\
\text { spp.(\%) }\end{array}$ & $\begin{array}{c}\text { Enterobact } \\
\text { er spp.(\%) }\end{array}$ & $\begin{array}{c}\text { Citrobacte } \\
\text { r spp.(\%) }\end{array}$ & $\begin{array}{c}\text { Acinetobact } \\
\text { er spp.(\%) }\end{array}$ \\
\hline Numbers & 18 & 3 & 1 & 6 \\
\hline AMP & 0 & 0 & 0 & 0 \\
\hline AC & 0 & O & O & $2(33.33)$ \\
\hline CU & 0 & 0 & $1(100.00)$ & $1(16.66)$ \\
\hline CE & 0 & 0 & $1(100.00)$ & $1(16.66)$ \\
\hline CI & $3(16.66)$ & 0 & $1(100.00)$ & $2(33.33)$ \\
\hline CO & $3(16.66)$ & 0 & $1(100.00)$ & $1(16.66)$ \\
\hline G & $2(11.10)$ & $1(33.30)$ & $1(100.00)$ & $3(50.00)$ \\
\hline CF & $5(27.80)$ & $1(33.30)$ & $1(100.00)$ & $3(50.00)$ \\
\hline IMP & $13(72.20)$ & $2(66.70)$ & $1(100.00)$ & $4(66.70)$ \\
\hline PT & $10(55.60)$ & $2(66.70)$ & $1(100.00)$ & $5(83.33)$ \\
\hline AK & $10(55.60)$ & $2(66.70)$ & 0 & $4(66.66)$ \\
\hline AO & $5(29.40)$ & $1(33.30)$ & $1(100.00)$ & $2(33.33)$ \\
\hline
\end{tabular}

\section{Discussion}

Neonatal sepsis is a leading cause of morbidity and mortality in developing countries. It is treatable if diagnosed on time. ${ }^{17}$ Clinical signs and symptoms of neonatal sepsis are not always specific therefore it is difficult to diagnose clinically. Knowledge about bacteriological profile and antibiogram play a important role in management of neonatal sepsis.

In our study male to female ratio is $1.75: 1$ and is comparable with studies done in Andhra Pradesh, Gujarat. ${ }^{18,19}$ Septicemia cases were more among males than females in almost all studies of neonatal sepsis. Male neonates are more affected compared to females, and could be because of a sex-linked genetic basis for host susceptibility. ${ }^{11}$

In present study, among culture positive sepsis cases, predominant risk factors were LBW $(66.40 \%)$ preterm $(45.31 \%)$ followed by PROM $(15.62 \%)$ and birth asphaxia (15.62\%). Comparable risk factors were found in study conducted in 2008 at kolkata. ${ }^{20}$ Study in 2005, at Manipal Reported prematurity followed by meconium aspiration syndrome as a risk factors. ${ }^{12}$ However study done in 2013 at Andhra pradesh reported inadequate antenatal care, assisted vaginal delivery, premature rupture of membranes and low birth weight were the important risk factors. ${ }^{18}$ Preterm and very low birth weight infants are vulnerable to nosocomial infections due to their immature immune systems, reduced placental passage of maternal antibodies, prolonged mechanical ventilation, prolonged hospitalization, use of indwelling catheters, endotracheal tubes, and other invasive procedures. ${ }^{9,21,22}$

Blood culture is considered as the "Gold standard" for the diagnosis of neonatal sepsis and it is necessary to do in all cases of clinically suspected septicemia. Bacteriological profile and their Antimicrobial sensitivity pattern play a vital role in management of these cases. ${ }^{23,6}$ Blood culture positivity in the present study is $64.64 \%$ and is comparable with the study done in 2011 at Pune. ${ }^{24}$ Study in 2005 at Manipal reported $16.40 \% .^{25}$ Study in 2010 at Bijapur reported $19.20 \%{ }^{26}$ Study in 2014 at Mangalore reported $46.66 \%{ }^{3}$ Study in 2012 at Gujarat reported $31.57 \%$ of culture positive cases. ${ }^{19}$ Few studies reported low culture yield, this may be because of fastidious organisms, maternal antibiotic treatment and small specimen volumes. ${ }^{27}$

In our study, in clinically suspected EOS cases 67 $(61.46 \%)$ were blood culture positive and in LOS 61 $(68.53 \%)$ were blood culture positive. Percentage of culture positivity was more in late onset septicemia as compared to early onset septicaemia. These findings could be because of the nosocomial (hospital acquired) or community acquired source of infection. Study in Raichur reported maximum culture positive cases were early onset septicemia as compared to late onset septicemia. According to the study ascending infections following rupture of membranes or the passage of the baby through the infected birth canal or resuscitation in the labour room were the reasons for early onset sepsis. ${ }^{28}$

Predominant organism isolated in the present study was Staphylococcus aureus (54.7\%) followed by Klebsiella spp. (14.1\%). Concordant results were found in other studies conducted in 2010 at Nepal, 2011 at Pune, 2014 at Tripura. $^{29,24,10}$ Higher incidence of Staphylococcus aureus could be because of the acquisition of pathogenic S.aureus from sites such as anterior nasal alae, axilla, perineal area of carriers; e.g., health workers, mother, relatives and umbilical stump of other babies was possible. The probable reason for Gram negative pathogens in new borns is due to the acquisition from the vaginal and fecal flora of the mother and the environment where the delivery occurs. ${ }^{19,2}$ National Neonatal-Perinatal database 
reported Klebsiella as the predominant (29\%) pathogen. ${ }^{30}$ Study in Nepal reported Staphylococcus aureus $(38.8 \%)$, Coagulase negative Staphylococci $(21.3 \%)$ as the commonest Gram positive isolates and among gram-negative organisms, Klebsiella species $(11.6 \%)$ and Enterobacter species $(9.7 \%)$ were the leading cause of neonatal sepsis. ${ }^{15}$ Another study conducted at bombay reported Enterobacter species along with Klebsiella spp. as predominant pathogens amongs Gram negative organisms and Staphylococcus aureus as the predominant isolate (79\%) among Gram positive isolates. ${ }^{23}$

In present study predominant organisms isolated in EOS were S. aureus, Candida spp., Enterococcus and Klebsiella spp. Results are comparable with study done in south India. ${ }^{31}$ Study done in New Delhi reported Klebsiella species $(35.5 \%)$ to be the most common etiological agent for EOS. ${ }^{13}$ The pathogens responsible for EOS generally reflect the predominant vaginal and fecal flora of the pregnant woman. ${ }^{32}$ In our study, the organisms causing early onset sepsis are very similar to those causing late onset sepsis which is comparable with Indian Scenario according to XXIV Annual Meeting of the National Neonatology Forum in which the organisms causing early onset neonatal sepsis were similar to those causing late onset neonatal sepsis. ${ }^{33}$

Vertical transmission from the mother and postnatal acquisition of infection from the surrounding environment has been suggested in literature for pathogenesis of neonatal septicaemia. In Western countries, Group B Streptococci (GBS) is leading cause for neonatal sepsis. ${ }^{19}$ In present study there was not a single case of neonatal septicemia by GBS. The spectrum of causative organisms for neonatal sepsis changes over time and varies from region to region. This could be because of changing pattern of antibiotic use and changes in lifestyle, ethnic and socioeconomic differences. ${ }^{34,19,35}$

In present study among Staphylococcus aureus, 43 (61.42\%) isolates were MRSA and most of them were sensitive only to vancomycin and linezolid. Most of the MSSA were sensitive to cotrimoxazole, clindamycin, ciprofloxacin, vancomycin and linezolid. Among CoNS, out of 7 isolates, 2 isolates were MRCoNS. Study done in New Delhi reported all the Gram positive cocci (GPC) were susceptible to vancomycin, linezolid and clindamycin while they were least susceptible to penicillin. ${ }^{2}$ Study done in 2010 at Nepal found most of the gram positive isolates showed susceptibility to commonly used aminoglycosides and quinolones. ${ }^{29}$ Another Study done in 2011 at Pune found that most of the MSSA(methicillin sensitive staphylococcus aureus) strains were sensitive to routinely used antibiotics and they are probably acquired from the community and MRSA strains, were possibly hospital acquired. ${ }^{24}$

In our study most of the Gram negative bacilli were sensitive to imipenem, piperacillin tazobactam \& amikacin. Results were comparable with study done in bijapur. ${ }^{25}$ Study in New delhi reported comparable sensitivity pattern along with high sensitive to colistin $\&$ meropenem. ${ }^{13}$ However study in Nepal reported better sensitivity to aminoglycosides (amikacin and gentamicin) and third generation cephalosporins. In general, the sensitivity of the Gram negative isolates to aminoglycosides supports continued use of the antibiotic in the initial, empiric treatment of neonatal sepsis in the hospital and also supports WHO recommendations that management of infants up to age 2 months include parenteral use of benzyl penicillin or ampicillin plus an aminoglycoside such as gentamicin. ${ }^{15}$

\section{Conclusion}

Due to changing spectrum of the causative agents of neonatal sepsis and their antimicrobial susceptibility patterns, a positive blood culture and the antimicrobial susceptibility testing of the isolates is important in choosing the appropriate antibiotic in treating the sepsis cases. Blood culture is the "Gold standard" For the diagnosis of neonatal septicaemia, and it is necessary to do in all cases of suspected septicemia. Preventive measures to reduce the load of neonatal sepsis are hand hygiene practice, barrier nursing, promotion of clean deliveries, exclusive breast feeding.

\section{References}

1. Bangi VA, Devi SS. Neonatal sepsis: A risk approach. $J$ NTR Univ Health Sci 2014;3:254-8.

2. Shah AJ, Mulla SA, Revdiwala SB. Neonatal sepsis: High antibiotic resistance of the bacterial pathogens in a neonatal intensive care unit of a tertiary Care hospital. $J$ Clin Neonatol 2012;1:72-5.

3. Nayak S, Rai R, Kumar VK, Sanjeev H, Pai A, Ganesh HR. Distribution of microorganisms in neonatal sepsis and antimicrobial susceptibility patterns in a tertiary care hospital. Arch Med Health Sci 2014;2:136-9.

4. A.L. Shane, B.J. Stol.1 Neonatal sepsis: Progress towards improved outcomes. J Infection 2014;68:S24-S32.

5. Barbara $\mathbf{J}$ stoll, Andi L shane, Infections of the neonatal infant, Nelson Text Book of Paediatrics, 20th edition, 1:909-25.

6. Rajendraprasad BM, Basavaraj KN, Antony B. Bacterial spectrum of neonatal septicemia with their antibiogram with reference to various predisposing factors in a tertiary care hospital in Southern India. Ann Trop Med Public Health 2013;6:96-9 .

7. Sharma P, Kaur P, Aggarwal A et al. Staphylococcus Aureus- The Predominant Pathogen in the Neonatal ICU of a Tertiary Care Hospital in Amritsar, India, J Clin Diagn Res 2013;7(1): 66-9.

8. Mondal SK, Nag DR, Bandyopadhyay R, Chakraborty D, Sinha SK. Neonatal sepsis: Role of a battery of immunohematological tests in early diagnosis. Int J App Basic Med Res 2012;2:43-7.

9. Mutlu M, Aslan Y, Saygin B et al. Neonatal Sepsis Caused by Gram-negative Bacteria in a Neonatal Intensive Care Unit: A Six Years Analysis. $H K J$ Paediatr (new series) 2011;16:253-257.

10. Debnath J, Das PK. Bacteriological profile and antibiotic susceptibility pattern of neonatal septicemia in a tertiary 
care hospital of Tripura. Ind J Microbiol Res 2015;2(4):238-43.

11. Gomella TC. Neonatology: Management, procedures, on call problems, diseases and drugs.2004; 5th edition: 43440.

12. Bhat $\mathrm{Y}$ et al.: Bacterial isolates of early-onset neonatal sepsis and their antibiotic susceptibility pattern between 1998 and 2004: an audit from a center in India. Italian J Pediatr 2011;37:32.

13. Jajoo M, Kapoor K, Garg LK, Manchanda V, Mittal SK. To study the incidence and risk factors of early onset neonatal sepsis in an out born neonatal intensive care unit of India. J Clin Neonatol 2015;4:91-5.

14. Forbes, Sahm, Weissfeld. Bailey and Scott's Diagnostic Microbiology. 13th edition China. 2014; chapter 68:860 77.

15. Shrestha P, Das BK, Bhatta NK et al. Clinical and Bacteriological profiles of blood culture positive sepsis in new borns. J Nepal Paediatr Soc 2007;27(2):64-7.

16. CLSI. Performance Standards for Antimicrobial Susceptibility Testing; Twenty-Fifth Informational Supplement. CLSI document M100-S25. Wayne, PA: Clinical and Laboratory Standards Institute; 2015

17. Makkar M, Gupta C, Pathak R, Garg S, Mahajan NC. Performance evaluation of hematologic scoring system in early diagnosis of neonatal sepsis. J Clin Neonatol 2013;2:25-9.

18. Bangi VA, Devi SS. Neonatal sepsis: A risk approach. $J$ NTR Univ Health Sci 2014;3:254-8.

19. Shah AJ, Mulla SA, Revdiwala SB. Neonatal sepsis: High antibiotic resistance of the bacterial pathogens in a neonatal intensive care unit of a tertiary Care hospital. $J$ Clin Neonatol 2012;1:72-5.

20. Mondal SK, Nag DR, Banyopadhyay R, Chakraborty D, Sinha SK. Neonatal sepsis-Role of certain immunohematologic tests in rapid diagnosis. J Acad Med Sci 2012;2:97-100.

21. Birju A Shah \& James F Padbury (2014) Neonatal sepsis, Virulence, 5:1, 170-178, DOI: 10.4161/viru.26906.

22. Gonzalez AC,Spearman P W, Stoll et al. Evaluation of Neonatal Sepsis. Pediatr Clin N Am 2013;60:367-89.

23. Mathur M, Shah H, Dixit K, Khambadkone S, Chakrapani A, Irani S. Bacteriological profile of neonatal septicemia cases (for the year 199091). J Postgrad Med 1994;40:1820.

24. Misra RN, Jadhav SV, Ghosh P, Gandham N, Angadi K, Vyawahare C.Role of sepsis screen in the diagnosis of neonatal sepsis. Med J DY Patil Univ 2013;6:254-7.

25. R Bhat $\mathrm{Y}$ and Rao A. the performance of haematological screening parameters and CRP in early onset neonatal infections. J Clin Diagn Res 2010;10(4):3331-6.

26. Jyothi P, Basavaraj MC, Basavaraj PV. Bacteriological profile of neonatal septicemia and antibiotic susceptibility pattern of the isolates. J Nat Sc Biol Med 2013;4:306-9.

27. Venkatesh M, Flores A, Luna RA et. Molecular microbiological methods in the diagnosis of neonatal sepsis. Expert Rev Anti Infect Ther 2010;8(9):1037-48.

28. Hegadi SS, Jitendranath A. Evaluation of risk factors and bacteriological profile of Neonatal Sepsis among patients admitted at Navodaya Medical College, Hospital and Research Center, Raichur. Ind J Microbiol Res 2017;4(3):298-304.

29. Narayan Gyawali \& Raj Kumari Sanjana, Bacteriological Profile and Antibiogram of Neonatal Septicemia, Ind $J$ Pediatr 2013;80(5):371-74.

30. Vital Statistics: Neonatal morbidity and mortality; report of the National neonatal-Perinatal database. Ind Pediatr 1997;34:1039-42.
31. Rao PS, Baliga M, Shivananda PG. Bacteriology of neonatal septicaemia in a rural referral hospital in south India. J Trop Pediatr 1993;39(4):230-3.

32. Ramesh Bhat. Y, Kumar N. Outcome of sepsis evaluations in very-low-birthweight premature neonates. J Clin Diagn Res 2009;3:1847-52.

33. Isaacs D. Neonatal Sepsis. The Antibiotic Crisis; Ind Pediatr 2005;42:9-13.

34. Rahman S Hameed A, Roghani MT et al. Multidrug resistant neonatal sepsis in Peshawar, Pakistan. Dis Child Fetal Neonatal Ed 2002;87:F52-F54.

35. Sundaram V, Kumar p, Dutta $S$ et al. Blood culture confirmed bacterial sepsis in neonates in a North Indian tertiary care centre: Changes over the last decade. Jpn J Infect Dis 2009;62:46-50. 